\title{
The angiotensin converting enzyme I/D polymorphism in Russian athletes
}

\author{
Igor B Nazarov*,1,3, David R Woods ${ }^{2}$, Hugh E Montgomery ${ }^{2}$, Olga V Shneider ${ }^{1,3}$, \\ Vasiliy I Kazakov ${ }^{1}$, Nikolai V Tomilin ${ }^{1}$ and Viktor A Rogozkin ${ }^{3}$
}

${ }^{1}$ Institute of Cytology of the Russian Academy of Sciences, Tikchoretski Ave. 4, Saint Petersburg, 194064, Russia; ${ }^{2}$ Department of Cardiovascular Genetics, 3rd floor Rayne Institute, University College London, 5 University Street, London WC1E 6JJ, UK; ${ }^{3}$ Saint-Petersburg Scientific Research Institute of Physical Culture, 2 Dinamo Street, Saint Petersburg, 197110, Russia

The deletion (D) allele of the human ACE gene is associated with higher ACE activity than the insertion (I) allele. There is controversy as to whether the ACE genotype may be associated with elite athletic status; recent studies have identified no significant associations amongst those drawn from mixed sporting disciplines. However, such lack of association may reflect the mixed nature of such cohorts, given that an excess frequency of the I allele has been reported amongst elite endurance athletes, and an excess of the $D$ allele amongst those engaged in more power-orientated sports. We examined this hypothesis by determining ACE I/D allele frequency amongst 217 Russian athletes (swimmers, skiers, triathletes and track-and-field participants) prospectively stratified by performance ('outstanding' or 'average'), and the duration of their event (SDA ( $<1 \mathrm{~min}$ ), MDA (1 to $20 \mathrm{~min}$ ), and LDA ( $>20 \mathrm{~min}$ ): short, middle and long distance athletes respectively). ACE genotype and allele frequencies were compared to 449 controls. ACE genotype frequency amongst the whole cohort, or the outstanding athletes alone, was no different to that amongst sedentary controls. However, there was an excess of the $D$ allele (frequency $0.72, P=0.001$ ) amongst the outstanding SDA group, and an excess of the $I$ allele (frequency $0.63, P=0.032$ ) amongst the outstanding MDA group. These findings were replicated in the outstanding swimmers, with track and field SDA similarly demonstrating an excess of the D allele $(P=0.01)$. There was no association found between the outstanding LDA and ACE genotype $(P=0.27)$. These data not only confirm an excess of the $D$ allele in elite SDA, and I allele in elite MDA, but also offer an explanation as to why any such association may be hard to detect amongst a heterogeneous cohort of mixed athletic ability and discipline. European Journal of Human Genetics (2001) 9, 797-801.

Keywords: angiotensin converting enzyme; ACE; athletes; sport

\section{Introduction}

A polymorphism in intron 16 of the human angiotensin Iconverting enzyme (ACE) gene has been identified in which the presence (insertion, I allele) rather than the absence (deletion, D allele) of a $287 \mathrm{bp}$ Alu-sequence insertion fragment is associated with lower serum ${ }^{1}$ and tissue ${ }^{2}$ ACE activity.

*Correspondence: Igor B Nazarov, Med. Biological Chemistry, 4107 Tupper Hall, One Shields Avenue, University of California at Davis, Davis, CA 95616, USA. Tel: +1 530 7525913; Fax: +1 530 7523516;

E-mail: i_naz@mail.ru

Received 2 April 2001; revised 28 June 2001; accepted 5 July 2001
An excess of the I allele has been associated with some aspects of endurance performance, being identified in elite British distance runners ${ }^{3}$ and mountaineers. ${ }^{4}$ In addition, an excess of the I allele is present in Australian ${ }^{5}$ and Croatian ${ }^{6}$ rowers as well as Spanish elite athletes. ${ }^{7}$ Conversely, an excess of the $\mathrm{D}$ allele has been reported amongst elite athletes in more power-oriented events such as short distance swimming $^{3,8}$ and sprinting. ${ }^{3}$

However, there has been debate as to the reproducibility of such associations. Several studies have failed to identify any association with elite endurance performance. ${ }^{9-11}$ Taylor et $a l^{9}$ examined hockey players, cyclists, skiers, track and field athletes, swimmers, rowers, gymnasts and 'others'. Similarly, 
the mixed cohort examined by Karjalainen ${ }^{10}$ included diverse sports such as long distance running, orienteering, cross country skiing and triathlon. The 192 athletes studied by Rankinen et l $^{11}$ also included skiers, long and middle distance runners, cyclists and biathletes (the latter would also have to to demonstrate more than a proficiency at rifle marksmanship). Such studies thus comprise individuals selected from diverse sporting disciplines, with potential variation in standard, with events of varying duration and skill mix.

In general, therefore, the association of ACE genotype with sporting prowess is recognised in studies of elite athletes drawn from a single sporting discipline, ${ }^{3-6,8}$ in whom the I and $\mathrm{D}$ alleles seems to associate with some aspects of endurance and power performance respectively. ${ }^{3-6,8}$ The use of subjects from mixed disciplines might thus account for the lack of association reported in such study groups. ${ }^{9-11}$

We have tested this hypothesis in the study of a mixed cohort of Russian athletes. ACE gene I/D allele frequencies for the cohort overall were first compared to those in a control sample. Subsequently, allele frequency was compared across event duration in individual sporting disciplines, for those performing at the highest level.

\section{Materials and methods}

The University of St Petersburg Ethics Committee approved the study and written informed consent was obtained from each participant.

\section{Subjects and controls}

Two hundred and seventeen male and female Russian athletes of regional or national competitive standard were recruited from the following sports: swimming ( $n=66)$, trackand-field athletics $(n=81)$, cross-country skiing $(n=52)$, and the triathlon $(n=18)$.

Our a priori intention was to clarify whether previously documented associations between the ACE polymorphism and elite athletes found in the study of a single sporting discipline could be duplicated in Russian athletes. Secondly, we wanted to determine whether negative studies that have found no association were due to the combining of athletes from different sports with different elements of power/endurance (anaerobic/aerobic) activity. The athletes were therefore prospectively stratified into groups according to event duration, covering a spectrum from the more 'power'-oriented to the more endurance-oriented. SDA (short distance athletes) consisted of 'sprinters', performing their given task in under $1 \mathrm{~min}$ (predominantly anaerobic energy production). MDA (middle distance athletes) were those competing over 1 to $20 \mathrm{~min}$ (mixed anaerobic and aerobic energy production), and LDA (long distance athletes) were those performing over more than $20 \mathrm{~min}$ (aerobic). The athletes were further classified by their standard of competition based on previous performances.
'Outstanding' athletes $(n=141)$ were at least national representatives. This group included 81 European and Russian champions and 19 Olympic or World champions. 'Average' athletes $(n=76)$ were regional competitors with no less than 7 years experience participating in their sport, but who had never been selected for the national team. This may have introduced unintended selection bias but was fundamental to the study aim. In order to further avoid selection bias, three methods were used to recruit the outstanding athletes; targeting of national teams, information provided by national coaching staff, and athletes attending national training camps.

Controls consisted of 449 healthy unrelated volunteers (111 students of St Petersburg University, aged 18-20, and 338 blood donors, age 25-45). The athletes and control groups were all Caucasian Russians, with an equivalent ratio from European and Siberian descent (3:1 in both groups). Further characteristics are presented in Table 1.

\section{Genotyping}

DNA was extracted from white blood cells or mouthwash samples as previously described. ${ }^{12}$ ACE genotype was determined using a three-primer method, ${ }^{13}$ yielding amplification products of $65 \mathrm{bp}$ (I allele) and $84 \mathrm{bp}$ (D allele). These were separated by electrophoresis on a $7.5 \%$ polyacrylamide gel and visualised using ethidium bromide. Genotyping was performed by experienced staff blind to subject data.

\section{Statistical analysis}

Allele frequencies were determined by gene counting. Genotype distribution and allele frequencies between groups of athletes and controls were then compared by $\chi^{2}$ test. $P$ values of $<0.05$ were considered statistically significant. By $a$ priori hypothesis, primary independent analyses explored differences in allele frequency between mixed athletes and controls, and by event duration in truly elite athletes competing in sports in which an association had been previously identified.

Table 1 ACE genotype distribution of the athletes and controls with sex (frequencies) and age (average $\pm S E$ )

\begin{tabular}{lccc}
\hline & \multicolumn{3}{c}{ ACE genotype } \\
& \multicolumn{1}{c}{ ID } & $D D$ \\
\hline Athletes & & & \\
All, $n=217$ & 0.19 & 0.51 & 0.29 \\
Male, $n=141$ & 0.20 & 0.54 & 0.26 \\
Female, $n=76$ & 0.18 & 0.46 & 0.36 \\
Age, years & $28 \pm 14$ & $30 \pm 17$ & $28 \pm 16$ \\
Sport experience, years & $15 \pm 11$ & $16 \pm 11$ & $14 \pm 10$ \\
Controls & & & \\
All, $n=449$ & & & \\
Male, $n=269$ & 0.23 & 0.52 & 0.24 \\
Female, $n=180$ & 0.22 & 0.54 & 0.24 \\
Age, years & 0.26 & 0.49 & 0.25 \\
& $32 \pm 12$ & $32 \pm 10$ & $33 \pm 10$ \\
\hline
\end{tabular}




\section{Results}

The subjects' age, male/female ratio and years in their chosen sport did not differ by ACE genotype (Table 1). ACE genotype distributions amongst subjects and controls were in Hardy-Weinberg equilibrium, being similar to that in other reported groups. ${ }^{3-11}$ As hypothesised, the genotype distribution and allele frequency amongst the whole cohort (19.3\% II, 51.2\% ID, 29.5\% DD, D allele frequency 0.55$)$, was similar to that amongst sedentary controls (23.4\% II, 52.3\% ID, and $24.3 \%$ DD, D allele frequency $0.50 ; P=0.27$, and 0.11 , for genotype and allele frequency respectively). Similarly, the whole cohort of 'outstanding' athletes (20.6\% II, 53.9\% ID, 25.5\% DD, D allele frequency 0.50 ) were no different to controls $(P=0.78$, and 0.55 , for genotype and allele frequency respectively). However, when only outstanding athletes were considered, ACE genotype was indeed associated with event duration: an excess of $\mathrm{D}$ alleles $(P=0.001)$ being noted in the SDA group, and an elevated frequency of the I allele in the MDA $(P=0.032)$ group (Figure 1$)$.

In considering individual sporting disciplines (Table 2 ), the mixed cohort of average and elite swimmers again demonstrated no association with the ACE genotype $(P=0.67)$. However, again examining allele frequencies by duration of event, a significant excess of the $\mathrm{D}$ allele was evident in the SDA $(P=0.042)$, with an excess of the I allele in the MDA $(P=0.042)$. As previously reported these findings were confined to the elite swimmers and were not replicated amongst the average athletes.

The track and field athletes demonstrated an excess of the D allele in outstanding SDA $(P=0.01)$, with an excess of the DD genotype $(P=0.018)$, which was not present in the average

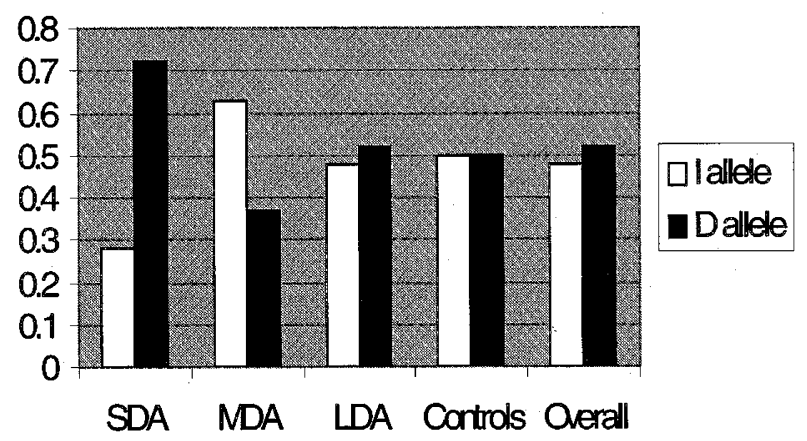

Figure 1 I and D allele frequencies in 141 outstanding Russian athletes, by duration of event, and sedentary controls. As hypothesised, the overall cohort of 'outstanding' athletes failed to demonstrate any association with the ACE genotype $(P=0.78$, and 0.55 by $\chi^{2}$ test compared to controls, for genotype and allele frequency respectively). However, ACE genotype was associated with event duration: an excess of $D$ alleles $(P=0.001)$ being noted in the SDA (short-distance athletes) group, and an elevated frequency of the I allele in the MDA (middle-distance athletes) group $(P=0.03)$.

Table 2 ACE genotype distribution and frequencies of ACE gene D allele in athletes stratified by sporting discipline, standard and duration of event. Comparison with controls was by $\chi 2$ test

\begin{tabular}{|c|c|c|c|c|c|c|c|c|c|c|c|c|}
\hline \multirow[b]{3}{*}{ Sport } & \multirow[b]{3}{*}{ Level } & \multirow[b]{3}{*}{ Group } & \multirow[b]{3}{*}{$\mathrm{n}$} & \multicolumn{4}{|c|}{ Genotype } & \multicolumn{5}{|c|}{$D$ allele } \\
\hline & & & & & & & & & & & & \\
\hline & & & & $\mathrm{n}$ & fr. & $\mathrm{n}$ & fr. & $\mathrm{n}$ & fr. & $\mathrm{P}$ value & fr. & P value \\
\hline \multirow[t]{4}{*}{ Swimming } & All & All & 66 & 16 & 0.24 & 31 & 0.47 & 19 & 0.29 & 0.667 & 0.52 & 0.695 \\
\hline & & SDA & 8 & 1 & 0.13 & 3 & 0.38 & 4 & 0.50 & 0.242 & 0.69 & 0.147 \\
\hline & Outstanding & LDA & 12 & 3 & 0.25 & 7 & 0.58 & 2 & 0.17 & 0.829 & 0.46 & 0.656 \\
\hline & & MDA & 24 & 9 & 0.38 & 13 & 0.54 & 2 & 0.08 & 0.113 & 0.35 & $0.042^{*}$ \\
\hline \multirow[t]{7}{*}{ Track-and-Field } & All & All & 81 & 13 & 0.16 & 38 & 0.47 & 30 & 0.37 & $0.043^{*}$ & 0.60 & $0.018^{* *}$ \\
\hline & Average & LDA & 3 & 2 & 0.67 & 1 & 0.33 & 0 & 0.00 & 0.193 & 0.17 & 0.099 \\
\hline & & MDA & 20 & 3 & 0.15 & 9 & 0.45 & 8 & 0.40 & 0.262 & 0.63 & 0.136 \\
\hline & & SDA & 27 & 3 & 0.11 & 14 & 0.52 & 10 & 0.37 & 0.190 & 0.63 & 0.174 \\
\hline & Outstanding & LDA & 10 & 2 & 0.20 & 5 & 0.50 & 3 & 0.30 & 0.910 & 0.55 & 0.687 \\
\hline & & MDA & 7 & 2 & 0.29 & 4 & 0.57 & 1 & 0.14 & 0.821 & 0.43 & 0.573 \\
\hline & & SDA & 14 & 1 & 0.07 & 5 & 0.36 & 8 & 0.57 & $0.018^{* *}$ & 0.75 & $0.010^{* *}$ \\
\hline Triathlon & Outstanding & LDA & 18 & 2 & 0.11 & 14 & 0.78 & 2 & 0.11 & 0.105 & 0.50 & 0.958 \\
\hline Totals & & & 217 & 42 & 0.19 & 111 & 0.51 & 64 & 0.29 & 0.266 & 0.55 & 0.113 \\
\hline Controls & & & 449 & 105 & 0.23 & 235 & 0.52 & 109 & 0.24 & 1.000 & 0.50 & 1.000 \\
\hline
\end{tabular}

${ }^{*} P<0.05,{ }^{* *} P<0.02$. SDA, short distance athletes; MDA, middle distance althletes; LDA, long distance athletes. 
athletes. These were responsible for the significant excess of the DD genotype $(P=0.043)$, and D allele $(P=0.018)$, in track and field athletes overall.

There was no relationship with ACE genotype amongst the skiers and triathletes.

\section{Discussion}

These data confirm our hypothesis that the study of a mixed cohort of athletes from various sporting disciplines, or the inclusion of non-elite athletes, may result in failure to demonstrate an association between elite athletes and the ACE genotype. However, in considering only outstanding athletes by duration of event (and hence relative contribution of anaerobic/power component compared to aerobic/endurance), we confirm an excess of the $\mathrm{D}$ allele in the SDA with an excess of the I allele in the MDA. Such findings are consistent with those previously reported. $^{3-6,8}$

Statistical adjustment for multiple comparison was not required in this study, as separate primary analysis for a single gene were performed according to a priori criteria, which aimed to confirm previously-identified associations.

It is interesting that we failed to demonstrate an excess of the I allele in the outstanding long-distance athletes, and hence did not find an increasing linear trend of I allele with increasing aerobic component as has previously been described. $^{3}$ This may relate to differences in categorising athletes; Myerson et $\mathrm{al}^{3}$ examined allele frequency by distance; $200 \mathrm{~m}, 400-3000 \mathrm{~m}$, and symbol $\geqslant 5000 \mathrm{~m}$, whereas in this study we categorised athletes by duration of event (under $1 \mathrm{~min}, 1$ to 20 , and over $20 \mathrm{~min}$ ). This was done to enable reasonable comparison between outstanding athletes of different disciplines and in order to account for the different times taken to complete the same distance. An elite cross country skier will complete the $5000 \mathrm{~m}$ in around $12 \mathrm{~min}$, an elite $5000 \mathrm{~m}$ runner in around 13 (current world record $12 \mathrm{~m}$ in $39 \mathrm{~s}$ ). However, a swimmer will have only completed less than $1500 \mathrm{~m}$ by similar time points (the current swimming world $1500 \mathrm{~m}$ record being $14 \mathrm{~min} 41 \mathrm{~s}$ ). Hence, a $5000 \mathrm{~m}$ runner or skier would be categorised as a MDA by our criteria, but a long distance competitor by the criteria of Myerson et al. ${ }^{3}$ Indeed, only the symbol $\geqslant 5000 \mathrm{~m}$ runners reported by Myerson et al showed an excess of I alleles compared to controls, ${ }^{3}$ which equates to our similar finding in outstanding MDA (I allele frequency 0.62 and 0.63 , respectively). Secondly, there was no excess of I alleles in symbol $\geqslant 5000 \mathrm{~m}$ runners when compared to middle distance athletes in the cohort of Myerson et al. ${ }^{3}$ The trend of rising I allele frequency, from short to longer distance, partly reflects an excess of the $\mathrm{D}$ allele in anaerobic events, which we have confirmed.

Important additional findings include the excess of $\mathrm{D}$ alleles amongst only the outstanding SDA swimmers, and track and field athletes, with an excess of the I allele in the former elite, but not the average, MDA. This emphasises the need to examine outstanding athletes with stratification by the nature and duration of the event.

The lack of an association between ACE genotype and elite skiers and triathletes may help explain the failure to find an excess of the I allele in other cohorts $^{9-11}$ which have included a substantial proportion of such athletes. I alleleassociated endurance may be an important, but not prime, determinant of success in cross-country skiing and the triathlon. A cohort such as that previously reported, ${ }^{9}$ which includes swimmers and track and field athletes, without deference to event duration, is even less likely to demonstrate an ACE genotype effect, especially in light of the excess of D alleles in short distance swimmers and track and field athletes both in this study, and others. ${ }^{3,8}$

Although the $\mathrm{D}$ allele has been associated with greater training-related changes in left ventricular growth ${ }^{14}$ and $\mathrm{VO}_{2}$ max rise ${ }^{15}$ the mechanism underlying the association of the $\mathrm{D}$ allele with power oriented, anaerobic sports is most likely mediated through differences in skeletal muscle strength gain. A greater training-related increase in quadriceps muscle strength has been associated with the D allele ${ }^{16}$. Such effects may, in turn, depend upon increased ACE-mediated activation of the growth factor angiotensin $\mathrm{II}^{17,18}$ and increased degradation of growth-inhibitory bradykinin. ${ }^{19,20}$ Conversely, the I allele may influence endurance performance through improvements in substrate delivery ${ }^{21}$ and the efficiency of skeletal muscle, ${ }^{22}$ with subsequent conservation of energy stores. ${ }^{23}$

The II genotype is strongly associated with lower ACE activity, ${ }^{1,2}$ perhaps caused by an Alu associated transcription silencer, ${ }^{24,25}$ or by an unidentified polymorphic variant of the ACE gene promoter in linkage disequilibrium with the I allele $^{26}$. Indeed, the gene for growth hormone lies in close proximity. Although linkage to the ACE I/D polymorphic site seems unlikely, ${ }^{27,28}$ a physiological interaction seems possible as elevated angiotensin II stimulates growth hormone release in humans ${ }^{29}$ via the angiotensin II type 1 receptor. ${ }^{30}$

In conclusion, these data support the suggestion that subsequent studies might best focus upon only truly elite athletes, that only those of a single sporting discipline should be considered, and that cohort stratification should be undertaken in some fashion according to anaerobic/aerobic or power/endurance component, as has been previously described. ${ }^{3,8}$ The ACE I/D polymorphism should not be considered a 'gene for human performance', but a marker of modulation such that one would expect association only in the truly elite athlete according to the nature of the specific event.

\section{Acknowledgements}

We thank Valentina Saburova for technical assistance. This work was supported by grants from the Russian Fund for Basic Research 00-0404003, 01-04-49486. 


\section{References}

1 Rigat B, Hubert C, Alhenc-Gelas F, Cambien F, Corvol P, Soubrier F. An insertion/deletion polymorphism in the angiotensin-1converting enzyme gene accounting for half the variance of serum enzyme levels. J Clin Invest 1990; 86: 1343 -1346.

2 Danser AHJ, Schalekamp MADH, Bax WA et al. Angiotensin converting enzyme in the human heart: effect of the deletion/ insertion polymorphism. Circulation 1995; 92: 1387-1388.

3 Myerson S, Hemingway H, Budget R, Martin J, Humphries S, Montgomery $\mathrm{H}$. Human angiotensin I-converting enzyme gene and endurance performance. J Appl Physiol 1999; 87: 1313 1316.

4 Montgomery HE, Marshall RM, Hemingway H et al. Human gene for physical performance. Nature 1998; 393: 221-222.

5 Gayagay G, Yu B, Hambly B et al. Elite endurance athletes and the ACE I allele - the role of genes in athletic performance. Hum Genet 1998; 103: 48-50.

6 Jelakovic B, Kuzmanic D, Milicic D et al. Influence of angiotensin converting enzyme (ACE) gene polymorphism and circadian blood pressure (BP) changes on left ventricle (LV) mass in competitive oarsmen. Am J Hypertens 2000; 13: $182 \mathrm{~A}$.

7 Alvarez R, Terrados N, Ortolano R et al. Genetic variation in the renin-angiotensin system and athletic performance. Eur J Appl Physiol 2000; 82: 117-120.

8 Woods D, Hickman M, Jamshidi Y et al. Elite swimmers and the D allele of the ACE I/D polymorphism. Hum Genet 2001; 108: $230-232$.

9 Taylor RR, Mamotte CDS, Fallon K, Bockxmeer FM. Elite athletes and the gene for angiotensin-converting enzyme. J Appl Physiol 1999; 87: 1035 - 1037.

10 Karjalainen J, Kujala UM, Stolt A et al. Angiotensinogen Gene M235T polymorphism predicts left ventricular hypertrophy in endurance athletes. J Am Coll Cardiol 1999; 34: 494-499.

11 Rankinen T, Wolfarth B, Simoneau J et al. No association between the angiotensin-converting enzyme ID polymorphism and elite endurance athlete status. J Appl Physiol 2000; 88: $1571-1575$.

12 Bolla MK, Haddad L, Humphries SE, Winder AF, Day INM. A method for determination of hundreds of APOE genotypes utilising highly simplified, optimised protocols and restriction digestion analysis by microtitre array diagonal gel electrophoresis (MADGE). Clin Chem 1995; 41: 1599-1604.

13 O'Dell SD, Humphries SE, Day INM. Rapid methods for population-scale analysis for gene polymorphisms: the ACE gene as an example. Br Heart J 1995; 73: 368 - 371.

14 Montgomery HE, Clarkson P, Dollery CM et al. Association of Angiotensin-Converting Enzyme Gene I/D polymorphism with change in left ventricular mass in response to physical training. Circulation 1997; 96: 741-747.
15 Rankinen T, Perusse L, Gagnon J et al. Angiotensin-converting enzyme ID polymorphism and fitness phenotype in the HERITAGE Family Study. J Appl Physiol 2000; 88: 1029-1035.

16 Folland J, Leach B, Little T et al. Angiotensin-converting enzyme genotype affects the response of human skeletal muscle to functional overload. Exp Physiol 2000; 85: 575-579.

17 Brown NJ, Blais C, Gandhi SK, Adam A. ACE Insertion/Deletion Genotype Affects Bradykinin Metabolism. J Cardiovasc Pharmacol 1998; 32: $373-377$.

18 Liu Y, Leri A, Li B et al. Angiotensin II stimulation in vitro induces hypertrophy of normal and postinfarcted ventricular myocytes. Circ Res 1998; 82: $1145-1159$.

19 Murphey LJ, Gainer JV, Vaughan DE, Brown NJ. Angiotensinconverting enzyme insertion/deletion polymorphism modulates the human in vivo metabolism of bradykinin. Circulation 2000; 102: 829-832.

20 Linz W, Scholkens BA. A specific B2-bradykinin receptor antagonist HOE 140 abolishes the antihypertrophic effect of ramipril. Br J Pharmacol 1992; 105: 771-772.

21 Woods DR, Humphries SE, Montgomery HE. The ACE I/D Polymorphism and Human Physical Performance. Trends Endocrinol Metab 2000; 11: 416-420.

22 Williams AG, Rayson MP, Jubb M et al. The ACE gene and muscle performance. Nature 2000; 403: 614

23 Montgomery $\mathrm{H}$, Clarkson $\mathrm{P}$, Barnard $\mathrm{M}$ et al. Angiotensinconverting-enzyme gene insertion/deletion polymorphism and response to physical training. Lancet 1999; 353: 541-545.

24 Tomilin NV, Iguchi-Ariga SM, Ariga H. Transcription and replication silencer element is present within conserved region of human Alu repeats interacting with nuclear protein. FEBS Lett 1990; 263: 69-72.

25 Tomilin NV. Control of genes by mammalian retroposons. Int Rev Cytol 1999; 186: 1-48.

26 Rieder MJ, Taylor SL, Clark AG, Nickerson DA. Sequence variation in the human angiotensin converting enzyme. Nat Genet 1999; 22: $59-62$.

27 McKenzie CA, Julier C, Forrester T et al. Segregation and linkage analysis of serum angiotensin I-converting enzyme levels: evidence for two quantitative trait loci. Am J Hum Genet 1995; 57: $1426-1435$

28 Jeunemaitre X, Lifton RP, Hunt SC, Williams RR, Lalouel JM. Absence of linkage between the angiotensin converting enzyme locus and essential hypertension. Nat Genet 1992; 1: $72-75$.

29 Messerli FH, Nowaczynski W, Honda M et al. Effects of angiotensin II on steroid metabolism and hepatic blood flow in man. Circ Res 1977; 40: 204-207.

30 Coiro V, Volpi R, Capretti L et al. Stimulation of ACTH and GH release by angiotensin II in normal men is mediated by the AT1 receptor subtype. Regul Pept 1998; 74: 27 -30. 